\title{
Validación de la escala de evaluación para la prevención del dopaje en escolares (CUPIAD) Validation of the scale of assessment for the prevention of doping in school (CUPIAD)
}

*Javier Álvarez Medina, **Pedro Manonelles Marqueta, *Enrique Oliete Blanco, *Víctor Murillo Lorente, ***Alberto Nuviala Nuviala

\author{
*Universidad de Zaragoza (España), **Universidad Católica de Murcia (España), ***Universidad Pablo de Olavide (España)
}

Resumen. El dopaje es un término relacionado con el deporte y hoy en día afecta a todos sus niveles, siendo utilizado cada vez, en mayor medida, por personas más jóvenes. Los estudios muestran que existe un gran desconocimiento del mismo y sus efectos sobre la salud, lo que provoca que no haya un rechazo total y rotundo al uso de sustancias dopantes. En la actualidad, el consumo de sustancias dopantes se está usando fuera del deporte con otros objetivos siendo principalmente la mejora de la imagen corporal. Los instrumentos creados para saber los conocimientos, creencias y actitudes sobre el mismo han sido diversos, pero ninguno que se adapte a la población escolar española. El objetivo de este estudio ha sido validar un instrumento que permita conocer los conocimientos, creencias y actitudes que tienen los escolares españoles en materia de dopaje. La población de estudio está formada por 540 escolares de $1^{\circ}$ de la ESO de diferentes escuelas, todas ellas de la Comunidad Autónoma de Aragón y más concretamente de la ciudad de Zaragoza en el Noreste de España. Se realizó un análisis estadístico de los ítems, un análisis factorial exploratorio, un análisis factorial confirmatorio, pruebas de invarianza factorial, así como pruebas de fiabilidad y validez. Los resultados garantizan la validez y fiabilidad del instrumento denominado Cuestionario Programa de Intervención Antidopaje (CUPIAD) para medir los conocimientos, creencias y actitudes que tienen los jóvenes españoles sobre el dopaje, compuesto por 17 ítems agrupados en 5 dimensiones.

Palabras clave: dopaje, sustancias prohibidas, conocimientos, jóvenes, prevención

Abstract. Doping is a term related to sport and today affects all levels increasingly being used to a greater extent by younger people. Studies show that there is great ignorance of it and its effects on health, causing no full and resounding rejection of the use of doping substances. Currently, the use of doping substances is being used outside the sport with other objectives being mainly improving body image. The instruments created to know the knowledge, beliefs and attitudes about it have been different, but none that fits the Spanish school population. The aim of this study was to validate a tool to determine the knowledge, beliefs and attitudes that have Spanish schools on doping. The study population consists of 540 students of 1 st ESO different schools. a statistical analysis of the items, an exploratory factor analysis, confirmatory factor analysis, factorial invariance tests and tests of reliability and validity was performed. The results guarantee the validity and reliability of the instrument called CUPIAD to measure knowledge, beliefs and attitudes that young Spaniards have on doping, consisting of 17 items grouped into 5 dimensions.

Key words: doping prohibited substances, knowledge, youth, prevention.

\section{Introducción}

El dopaje es un problema actual que atenta contra la salud del deportista, siendo algunos de sus efectos sobre la salud irreversibles (Boulu, 2002; Reardon \& Creado, 2014). El dopaje destruye todos los valores educativos y formativos que tiene el deporte(World Antidoping Code, 2015; Lazuras, Barkoukis \& Tsorbatzoudis, 2015). En la actualidad ya no es un problema aislado del deporte profesional, afecta a todos sus ámbitos, incluyendo al deporte de ocio y al deporte escolar (Dvorak, Saugy, Pitsiladis \& Yannis, 2014). Como consecuencia de esta situación, la Agencia Mundial Antidopaje, en el artículo 18 y 19 del Código 2015 dentro del apartado «Educación e Investigación», estableció una vía de actuación contra el dopaje, «la social». Su objetivo es la prevención a través de programas de intervención educativa. Estos programas deben enseñar a los deportistas, entrenadores, personal de apoyo y a todas las personas relacionadas con la actividad física y el deporte, los peligros reales que encierran el uso de sustancias y métodos prohibidos (World Antidoping Code, 2015).

Distintos investigadores han estudiado y examinado los conocimientos y actitudes de los deportistas como punto de partida a posibles programas de intervención. Ozdemir et al. (2005) en su estudio realizado con 433 deportistas estableció que el 14,5\% de los deportistas han tomado alguna vez sustancias prohibidas, siendo las razones para su uso el aumento del rendimiento y resolver problemas de peso. Más de la mitad de los consumidores no tienen conocimientos de las reacciones adversas que pueden tener estas sustancias sobre su salud. Alaranta et al. (2006), en su estudio realizado con 446 deportistas becados por el Comité Olímpico Finlandés, estableció que el $90 \%$ consideraba que las sustancias y métodos prohibidos tienen efectos potenciadores del rendimiento. El 30\% afirmaba conocer a algún deportista que usa sustancias prohibidas y el 15\% admitió que le han ofrecido sustancias prohibidas.

Fecha recepción: 03-09-17. Fecha de aceptación: 02-02-17 Víctor Murillo Lorente victormurillo1301@hotmail.com
Otros autores han estudiado el fenómeno en jóvenes, como Laure, Lecerf, Friser \& Binsinger (2004) investigaron con una población de 1459 deportistas franceses con una edad en torno a 16 años y provenientes de centros de secundaria. Sus resultados demostraron que el $4 \%$ había consumido drogas al menos en una ocasión. Este consumo aumentaba con la edad y el nivel de competición, siendo mayor en los chicos. Un 7\% de deportistas aseguraba que el hecho de consumir no le suponía un riesgo continuo para la salud. Laure \& Binsinger (2005) en su estudio con atletas franceses adolescentes ( $n=6402)$, a través de un cuestionario autocompletado, confirmaron la problemática actual del dopaje en los jóvenes y establecieron que existe un mercado negro de fácil acceso para la obtención de estos productos al que son atraídos por otras personas.

En la misma línea, Skinner, Moston \& Engelberg (2012) realizaron una investigación con escolares australianos de 12 a 17 años, cuyo objetivo principal era identificar los factores que motivan a los jóvenes deportistas a usar sustancias dopantes para mejorar el rendimiento, al tiempo que se trataron de identificar los factores que disuaden a estos deportistas a usar las sustancias. El trabajo concluyó que existe un vínculo claro entre el funcionamiento moral y las actitudes hacia las sustancias prohibidas.

El consumo de sustancias prohibidas no es exclusivo entre jóvenes deportistas, sino que también se da en no deportistas (Laure et al., 2004), ya que sustancias que se usan como dopaje se están utilizando no sólo para mejorar el rendimiento deportivo (Bents, Tokish \& Goldberg, 2004) sino para mejorar la imagen desde un punto de vista estético (Ozdemir et al., 2005). Esta situación se convierte en más crítica todavía, puesto que autores como Peretti-Watel \& Lorente (2004) establecen una clara relación entre el consumo de drogas y el estilo de vida, al tiempo que avisan de la amenaza que supone el consumo de sustancias prohibidas por los adolescentes, drogas sociales como el tabaco y el alcohol, hace que la probabilidad de consumo de sustancias prohibidas, para aumentar o no el rendimiento, crezca considerablemente (Galán, Rodríguez-Aralejo, Díez-Gañán, Tobías, Zorrilla \& Gandarillas, 2006)

Existe un doble problema, por un lado, el propio uso de sustancias dopantes; por otra parte, el gran desconocimiento del dopaje y de sus 
efectos negativos sobre la salud, lo que seguramente impide que los jóvenes rechacen el consumo de sustancias dopantes (Laure et al., 2004).

En la investigación sobre los conocimientos, actitudes y creencias del dopaje se han utilizado diferentes instrumentos. Así, por ejemplo, Melia, Pipe \& Greenberg (1996), con una población de estudio de entre 11 y 18 años, utilizaron un cuestionario de 42 items. Stilger \& Yesalis (1999) diseñaron un instrumento de 50 ítems, siendo la población de estudio jugadores de fútbol americano de la escuela secundaria (16-18 años). Naylor, Gardner \& Zaichkowsky (2001) crearon una encuesta de 150 ítems que evalúa el consumo de sustancias ilícitas y no ilícitas de estudiantes americanos de secundaria, entre 16 y 18 años.

Otros autores, más recientemente, han realizado estudios con deportistas de diferentes edades y con adultos. Ozdemir et al. (2005) elaboraron un cuestionario de 24 ítems para determinar el conocimiento en materia de dopaje, su uso y conocer las principales razones para el consumo en deportistas turcos con una edad media de 21,8 \pm 3,7 años. Lorente, Peretti-Watel \& Grelot (2005) conformaron un cuestionario de 87 ítems que fue administrado a estudiantes de seis universidades de Ciencias del Deporte para conocer el uso de cannabis y su mejora en el rendimiento deportivo y no deportivo.

Junto a ellos, en la literatura se pueden encontrar otros trabajos que utilizan una metodologíamixta con el mismo objetivo, como el de Corvella (2010) que utilizó cuestionarios y entrevistas para analizar los conocimientos y actitudes de los deportistas ante el dopaje y su posible relación con las representaciones sociales en el deporte federado y aficionado o Butryn, Johson \& Masucci (2012) para saber los niveles de conocimientos sobre el dopaje en jóvenes deportistas de alto nivel y exprofesionales.

Para efectuar un estudio sobre los conocimientos, creencias y actitudes que los jóvenes españoles del Sistema Educativo tienen en materia de dopaje, las metodologías de estudio descritas anteriormente son poco apropiadas porque tienen una antigüedad considerable y la percepción social sobre el dopaje ha evolucionado mucho en los últimos años, además de que están dirigidos a poblaciones de estudio diferentes. Por lo tanto, para realizar un estudio como el descrito es necesario diseñar un cuestionario ad hoc que se ajuste plenamente a la población objeto de estudio.

Se ha decidido realizar este estudio en las clases de Educación Física porque este contexto es uno de los más utilizados para el análisis de los procesos que pueden mediar en los comportamientos morales de los adolescentes (Sánchez, et al., 2012; Ruiz Omeñaca, Ponce de León, Sanz \& Valdemoros, 2015), propiciando una educación integral del individuo (López Pastor, Pérez Brunicardi ManriqueArribas \& Mongas Aguado, 2016). La Educación Física se muestra como un lugar ideal para la educación en hábitos saludables (Méndez Alonso, Fernández Rio, Méndez Giménez \& Prieto Saborit, 2015). Educar en valores significa contribuir a la función integradora del individuo, incorporando prácticas y actitudes que den paso al cumplimiento de derechos y responsabilidades de las personas (Rodríguez, 2010).

Para su diseño, se siguen las recomendaciones de la AMA(World Antidoping Code, 2015) que establece que los programas antidopaje deben proteger lo intrínsecamente valioso del deporte, el denominado «Espíritu deportivo». De esta manera el tema del dopaje puede introducirse dentro del Sistema Educativo a través de las clases de Educación Física porque son las que mejor pueden transmitir el espíritu del deporte y los principios del juego limpio.

\section{Material y Método}

\section{Participantes}

Los participantes en este estudio fueron un total de 540 adolescentes matriculados en primer curso de Educación Secundaria Obligatoria durante el curso escolar 2014-15 de la Comunidad Autónoma deAragón, concretamente de la ciudad de Zaragoza en el Noreste de España. Niños fueron el 50.9\% y niñas el $49.1 \%$. Realizaban práctica deportiva de forma habitual un $67 \%$ del total.

\section{Instrumento}

La escala de evaluación para la prevención del dopaje en edad escolar desarrollada en este estudio denominada Cuestionario Programa de Intervención Antidopaje (CUPIAD) permite conocer los conocimientos, actitudes y creencias de los adolescentes en materia de dopaje. La versión preliminar de la escala, elaborada tras un trabajo cualitativo que sigue las normas establecidas por Carretero-Dios \& Pérez (2005) sobre la construcción de instrumentos de investigación, estuvo conformada por 22 ítems. Para la selección de los ítems, una vez definidas las dimensiones, se seleccionaron un listado de cuestiones en función de su relevancia en cuanto al contenido y a su viabilidad de aplicación, sin olvidar que son una concreción operativa de éstas (Rubio, Berg-Weger, Tebb, Lee \& Rauch, 2003). Con objeto de asegurar tanto la validez de contenido como la aplicabilidad del instrumento, esta primera versión fue sometida a un doble proceso de depuración: 1) juicio de expertos, donde ocho participantes analizaron la adecuación de los ítems con la dimensión que evaluaban mediante una escala Likert de cinco puntos, utilizándose el acuerdo inter-jueces para eliminar o depurar los ítems más problemáticos; 2) Estudio piloto de 30 participantes con el objeto de depurar los ítems que más problemas de comprensión generaban o que presentaban erratas en su formulación. De los 22 ítems iniciales, 17 superaron dicho proceso y fueron los que se utilizaron (tabla 1).

\section{Procedimiento}

Se consiguió el permiso del consejo de revisión de la Universidad. Posteriormente se informó a los responsables de los centros escolares que participaron en el estudio. Se solicitó permiso a los padres/madres del alumnado participante. Tras su aprobación se realizó la investigación. En el diseño se ha tenido en cuenta la normativa legal vigente española que regula protección de datos de carácter personal (Ley Orgánica 15/1999). También fueron respetados los principios fundamentales establecidos en la Declaración de Helsinki (revisión de 2013, Brasil). Antes de la realización del trabajo de campo, que se realizó mediante un cuestionario autoadministrado con presencia del encuestador, se obtuvo el consentimiento informado de los participantes. El tiempo invertido en la realización fue de unos 10 minutos.

\section{Análisis de datos}

En primer lugar se efectuó un análisis estadístico de los ítems. Se calculó media, desviación típica, asimetría, curtosis, coeficiente de correlación corregido ítem-total y fiabilidad si se elimina el ítem. Posteriormente se realizó un Análisis Factorial Exploratorio (AFE) mediante el análisis de componentes principales y rotación ortogonal Varimax. La adecuación de la matriz para realizar el AFE fue testada mediante el test Kaiser-Meyer-Olkin (KMO) y la Prueba de Esfericidad de Bartlett.

Se calculó posteriormente la fiabilidad del instrumento resultante mediante el coeficiente alfa de Cronbach. Como complemento a este índice de consistencia interna se calcularon otros dos indicadores derivados del análisis factorial como son la Theta (È) de Carmines (Carmines \& Zeller, 1979) y la Omega (Ù) de Heise \& Bohrnstedt (1970).

A continuación se realizó un análisis factorial confirmatorio. El método utilizado fue el de estimación robusta de máxima verosimilitud (ML). Para evaluar la bondad del ajuste, se revisaron los siguientes indicadores: $\chi 2$; $\chi 2 /$ gl; GFI; RMR; RMSEA; AGFI; TLI; CFI; IFI (Hair, Black, Babin, Anderson \& Tatham, 2006; Marôco, 2010). Además con el objeto de seguir las indicaciones de Byrne (2001) se adjunta el criterio de información de Aiken (AIC) y el índice de validación cruzada esperada (ECVI).

Para concluir se comprobó la validez discriminante. Este tipo de validez evalúa que el grado en una medida no se correlaciona con las medidas con las que se supone que tiene que diferir (Churchill, 1979). Para ello se han aplicado tres procedimientos diferentes: cálculo de correlaciones entre factores, estimación de modelos alternativos y construcción de los intervalos de confianza de la correlación de factores al 95\% de confianza.

Los análisis estadísticos de este trabajo se llevaron a cabo utilizando los paquetes estadísticos SPSS 21 y AMOS 21. 


\section{Resultados}

\section{Análisis estadístico de los ítems}

En la Tabla 1 se muestran los estadísticos descriptivos de losítems. Los valores de correlación ítem-total son iguales o superiores a .25. Se puede observar que los índices de asimetría y curtosis están por debajo del valor 1.96, lo que indica semejanza con la curva normal. Estos resultados permiten la utilización de técnicas factoriales que se realizarán a continuación. La fiabilidad de la escala evaluada con alfa deCronbach es de .751 .

del Análisis Factorial Exploratorio compuesto por 5 factores y 17 ítems, presentó valores correctos. Los índices de ajuste incremental fueron: AGFI=.945; TLI=.911; CFI=.929; IFI=.930. Los índices de ajuste de carácter absoluto fueron: $\mathrm{RMR}=.063$; RMSEA $=.037$; $\mathrm{GFI}=.961$. Finalmente AIC, ECVI y el cociente $\chi^{2} / \mathrm{gl}$ presentaron los siguientes valores: 276.053 ; .511 y 1.725 respectivamente.

\section{Validez discriminante}

La validez discriminante de la escala se constató por tres vías diferentes. La forma tradicional, mediante el cálculo de la matriz de correlaciones entre los factores de la escala. Como puede observarse en la Tabla 3, existió correlación significativa, positiva y moderada entre los factores que componen la escala, exceptuado el par Métodos-Consecuencias.

Actualmente se utiliza una versión modificada del procedimiento anterior, recomendada por Burnkrant \& Page (1982). Se trata de estimar modelos alternativos de tal forma que en cada uno de ellos se introduce como restricción que la correlación entre cada par de dimensiones es igual a 1 , y realizar con cada uno un test de diferencias de ji-cuadrado para comparar los modelos con el fin de evaluar si eran significativamente diferentes. En la Tabla 4 se comprueba como la diferencia entre los ji-cuadrado fue siempre significativa, por lo que las dimensiones de la escala fueron significativamente diferentes entre sí, corroborando la validez discriminante.

Como tercera vía para garantizar este tipo de validez, se han calculado todas las correlaciones posibles entre los factores, lo que ha permitido construir el intervalo de confianza de las correlaciones entre todas las dimensiones. Como se muestra en la Tabla 4, se puede confirmar la validez discriminante de la escala,

\section{Análisis de la estructura interna}

Para conocer la estructura factorial de la escala de evaluación para la prevención del dopaje en edad escolar, se realizó un análisis factorial exploratorio sobre los 17 ítems resultantes del análisis estadístico. Antes de realizar el análisis, se calculó la medida de adecuación muestral de Kaiser-Meyer-Olkin (KMO) y el test de esfericidad de Bartlett. El índice KMO mostró un valor de .751 y el test de Bartlett resultó estadísticamente significativo $\left(\chi^{136}\right.$ =1228,211; p > .001), lo que llevó a concluir que la aplicación del análisis factorial resultaba pertinente. Los factores conjuntamente explican un 50.13\% de la varianza (Tabla 2)

\section{Fiabilidad}

La fiabilidad del instrumento resultante fue medida con alpha de Cronbanch, obteniendo un valor de .751 para los 17 ítems. Como complemento a este índice de consistencia interna se calcularon otros dos indicadores derivados del análisis factorial como son la Theta (È) de Carmines(Carmines \& Zeller, 1979) y la Omega (Ù) de Heise \& Bohrnstedt (1970). Para los datos con los que hemos trabajado È alcanza un valor de .759 y Ù de 85 .

Análisis Factorial Confirmatorio

El modelo teórico hipotetizado resultante
Tabla 2 .

Estructura factorial rotada, comunalidades, autovalores, alfa de Cronbach y porcentaje de varianza explicada por cada factor

\begin{tabular}{|c|c|c|c|c|c|}
\hline Concepto & Utilidad & Métodos & $\begin{array}{l}\text { Deporte } \\
\text { y dopaje }\end{array}$ & $\begin{array}{c}\text { Origen } \\
\text { conducta }\end{array}$ & Extracción \\
\hline .706 & & & & & .557 \\
\hline .701 & & & & & .606 \\
\hline .717 & & & & & .549 \\
\hline .459 & & & & & .435 \\
\hline & .629 & & & & .418 \\
\hline & .640 & & & & .469 \\
\hline & .738 & & & & .569 \\
\hline & & .715 & & & .604 \\
\hline & & .742 & & & .583 \\
\hline & & & .575 & & .447 \\
\hline & & & .563 & & .384 \\
\hline & & & .673 & & .517 \\
\hline & & & .650 & & .429 \\
\hline & & & .598 & & .424 \\
\hline & & & .355 & & .247 \\
\hline & & & & .753 & .637 \\
\hline & & & & .723 & .647 \\
\hline 12.81 & 11.70 & 10.15 & 8.01 & 7.44 & 50.13 \\
\hline 3.50 & 1.55 & 1.24 & 1.13 & 1.08 & \\
\hline .658 & .614 & .581 & .643 & .563 & .751 \\
\hline
\end{tabular}


Tabla 3.

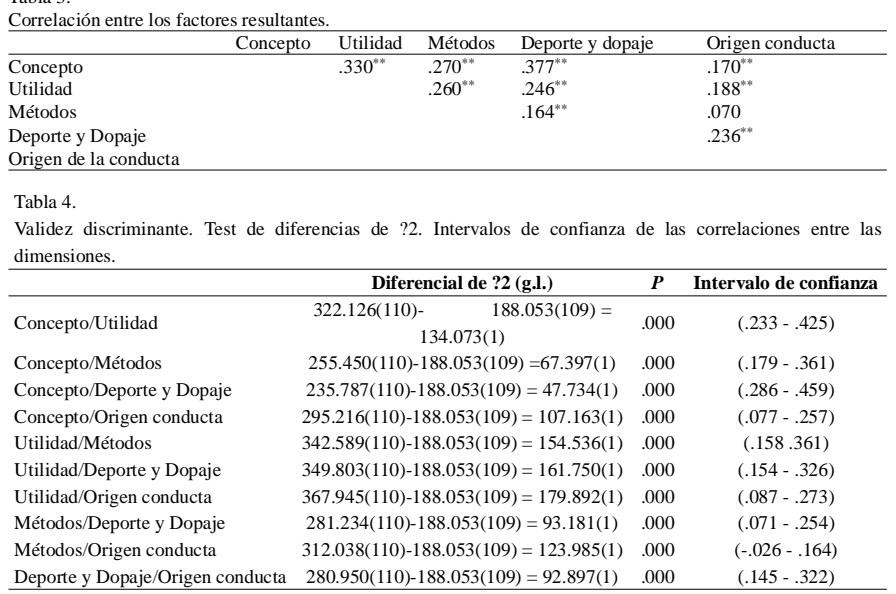

necesario contrastar que los ítems deben encontrarse relacionados entre sí, es decir, la matriz de correlaciones debe ser tal que puedan localizarse agrupamientos relevantes entre variables. Por ello es necesario realizar, antes de la aplicación del análisis, el cálculo de unos estimadores que aseguren que la matriz de correlaciones es la apropiada (Cortina, 1993), siendo las pruebas de elección la de esfericidad de Bartlett y el índice de Kaiser-Meyer-Olkin (KMO). El resultado de esta prueba dio la pertinencia del proceso al presentar un valor alto.

El análisis estadístico de los ítems y el posterior análisis factorial exploratorio dio como resultado cinco factores (Concepto; Utilidad; Métodos; Deporte y Dopaje; Origen de la conducta) que explican el $50.13 \%$ de la varianza, alrededor de los cuales se agrupaban los ítems.

El primero de los factores recoge información acerca del conocimiento que tienen los encuestados del término Dopaje. En los ítems de esta dimensión aparecen definiciones o explicaciones sencillas sobre el concepto y su ámbito de utiliza-

contiene el valor 1 al 95\% de confianza.

\section{Discusión}

El objetivo de este artículo es constatar la validez, fiabilidad y estructura factorial de la escala de evaluación para la prevención del dopaje en edad que permite conocer los conocimientos, actitudes y creencias de los adolescentes en materia de dopaje. Tras el diseño cualitativo de los ítems, según el procedimiento descrito por Carretero-Dios \& Pérez (2005), que tuvo tres momentos diferentes: diseño del instrumento; confección de un grupo de expertos para constatar la idoneidad y congruencia de los ítems; y estudio piloto; se procedió al análisis cuantitativo de los ítems resultantes. Este tratamiento tiene como fin garantizar la capacidad de poner de manifiesto las diferencias existentes entre los individuos. Por ello, el objetivo es conseguir un grupo de ítems que maximice la varianza del test, seleccionando aquellos con un elevado poder de discriminación, alta desviación típica y con puntuaciones medias de respuesta situadas en torno al punto medio de la escala (Bollen \& Long, 1993; Carretero-Dios \& Pérez, 2005; Nunnally \& Bernstein, 1995).

La puntuación media de los ítems estaba alrededor del punto medio de la escala (3.41 \pm 1.21$)$ y la desviación típica fue superior a 1, exceptuando un ítem, lo que demuestra la normalidad de los resultados según Carretero-Dios \& Pérez (2005). Además, los valores de asimetría y curtosis fueron inferiores a 2 lo que significa una distribución normal univariada de los datos (Bollen \& Long, 1993). Para calcular la discriminación de los ítems se recurrió al coeficiente de correlación corregido entre la puntuación en el ítem y la total. Este procedimiento buscaba aumentar la consistencia interna de la escala. Se consideraron adecuados valores mayores o iguales a .25 (Nunnally \& Bernstein, 1995). Con ello se pretendía suprimir aquellos ítems que generen respuestas demasiado unánimes y poco discriminativas (Streiner \& Norman, 1995).

Una vez que los ítems seleccionados pasaron los filtros iniciales, el objetivo siguiente era comprobar cómo se agrupaban empíricamente. En este momento la meta es explorar la estructura interna de la escala, su dimensionalidad o forma de agruparse los ítems (Elosua, 2003). Para comprobar la agrupación se utilizó el análisis factorial exploratorio. Dicho análisis proporcionó los agrupamientos de las variables que componen el cuestionario en función de criterios matemáticos basados en la correspondencia entre éstos para que posteriormente sean interpretados. El análisis factorial exploratorio sólo agrupa correlaciones similares, pero esta agrupación puede ser debida a más elementos que los propiamente conceptuales. El procedimiento de rotación utilizado fue Varimax, a pesar de que se aconseja para casos en que los factores no están relacionados. Se optó por este debido al interés teórico de separar, en la medida de lo posible, los factores resultantes, a pesar de constatar la relación de los factores (Carretero-Dios \& Pérez, 2007).

Previo a una correcta aplicación del análisis factorial exploratorio es ción. El segundo de los factores hace alusión a la utilidad que dicha conducta tiene: sirve para incrementar el rendimiento y evitar lesiones. El tercero de los factores, Métodos, hace alusión al conocimiento que los adolescentes tienen o no de posibles formas que los deportistas utilizan para doparse y de sus consecuencias sobre la salud. El factor Deporte y Dopaje incluye una serie de ítems relativos a agencias de lucha contra el Dopaje, casos y referencias de deportistas que se han dopado y cuestiones éticas deportivas. Finalmente, el factor Origen de la Conducta, aborda con sus ítems la relación motivos y resultados del Dopaje.

La consistencia interna de la escala fue medida mediante el alpha de Cronbanch, obteniendo un valor de .751. Como complemento a este índice de consistencia interna se calcularon otros dos indicadores derivados del análisis factorial como son la Theta (È) de Carmines (Carmines \& Zeller, 1979) y la Omega (Ù) de Heise \& Bohrnstedt (1970), siendo ambos coeficientes estimados superiores de alpha de Cronbanch. Los resultados obtenidos confirman el supuesto ád» è d» Ù, lo que demuestra una buena fiabilidad del instrumento.

La validez discriminante de la escala viene expresada por el contraste entre los diferentes factores que la componen. Si son realmente distintos los conceptos que la conforman y al mismo tiempo están relacionados, es entonces cuando se puede hablar de este tipo de validez (Lehmann, Gupta, \& Steckel, 1999). Para garantizarla se han calculado de diversas formas. La primera y tradicional, consiste en correlacionar los factores que componen la escala y comprobar que su relación sea significativa positiva y moderada. Los resultados obtenidos dan pie a afirmar este tipo de validez.

Solamente en la relación entre los factores: «Métodos» y «Origen de la conducta», no se ha encontrado una relación significativa. Este resultado puede explicarse desde el momento que los adolescentes a los que se les ha suministrado el instrumento, no cuentan con ningún tipo de formación en la temática que confirman la falta de formación y conocimiento sobre el dopaje que demuestran la mayoría de los estudios, independientemente del país donde se realizan (Laure et al., 2004; Boulu, 2002; Reardon \& Creado, 2014; Bodin \& González, 2010).

Bodin \& González (2010) utiliza una población deportista española y francesa formada por jóvenes entre 12 y 30 años que practican baloncesto, atletismo y ciclismo, así como entrenadores y padres. Llegan a las conclusiones de que el conocimiento sobre la prevención del dopaje entre los atletas, entrenadores y padres es muy escaso, dejando al deportista en una clara vulnerabilidad a cualquier tipo de influencia y la poca información que se tiene, proviene de los amigos y de los medios de comunicación (televisión, internet, prensa...) donde solo se habla del deporte de élite y donde las fuentes de origen de la información como mínimo son dudosas. Por ello, como es lógico, no relacionan los métodos del dopaje con el origen de la conducta en el deportista. Confirmando lo que dicen numerosos estudios, concluimos que se desconocen los efectos que puede tener el dopaje sobre la salud. 
Laure et al. (2004) con una muestra de 1459 escolares y una media de edad de 16 años, obtiene que un $7 \%$ de deportistas, asegura que el hecho de consumir no le supone un riesgo continuo para la salud, siendo esta respuesta más frecuente en los más jóvenes. Además, el 27\% opina que, con un adecuado control médico, la salud no tiene porqué correr peligro alguno.

Ozdemir et al (2005) encuentran que el 52,4\% de los consumidores no tienen conocimientos de las reacciones adversas que pueden tener estas sustancias sobre su salud. Bondarev, Galchinskiy, Ajitskiy \& Labskir (2009) con 844 estudiantes de la Universidad de Ucrania, con edades comprendidas de 18 a 23 años, obtienen que los estudiantes consideran que el dopaje no tiene un gran impacto negativo sobre la salud si se utiliza de manera razonable. Además, entre ellos es generalizada la opinión y creencia de que en pequeñas dosis y en proporciones razonables el dopaje puede ser beneficioso. Cordero SI, Cordero AT, Martínez, Belkys \& Cabrera (2014) también concluyen con una falta de formación que aparece en los jóvenes en materia de dopaje y sus efectos negativos.

En la actualidad también se comprueba este tipo de validez a través de otras dos vías. La primera es la propuesta por Burnkrant \& Page (1982), que trata de estimar modelos alternativos, de tal forma que en cada uno de ellos se introduce como restricción que la correlación entre cada par de dimensiones es igual a 1 , y realizar con cada uno un test de diferencias de ji-cuadrado para comparar los modelos con el fin de evaluar si eran significativamente diferentes. Los resultados han probado cómo la diferencia entre los ji-cuadrado fueron siempre significativas, por lo que las dimensiones de la escala de prevención del dopaje en edad escolar fueron diferentes entre sí, corroborando la validez discriminante.

La tercera y última de las vías consiste en calcular las correlaciones posibles entre los factores y construir los intervalos de confianza de las correlaciones entre todas las dimensiones. Los resultados, también han mostrado este tipo de validez, ya que ninguno de los intervalos de confianza de esas correlaciones contiene el valor 1 al 95\% de confianza (Anderson \& Gerbing, 1988)

\section{Conclusiones}

El instrumento ad hoc (CUPIAD) diseñado para medir los conocimientos, actitudes y creencias sobre el dopaje en jóvenes de 12-13 años dentro del Sistema Educativo ha demostrado ser válido y fiable. Sin embargo, no deja de ser un inicio sobre este tema.

\section{Limitaciones de estudio}

Las limitaciones en relación al número de alumnos utilizados en el trabajo de campo, así como la falta de bibliografía relativa al tema, hace que sea necesario seguir profundizando en el tema y mejorar, si es posible, el instrumento.

\section{Referencias}

Alaranta, A., Alaranta, H., Holmila, J., Palmu, P., Pietila K. \& Helenius, I. (2006). Self-reported attitudes of elite athletes towards doping: Differences between types of sport. Int J Sports Med, 27 (10), 842-846.

Anderson, J. C. \& Gerbing, D. W. (1988). Structural Equation Modeling in Practice: A Review and Recommended Two-Step Approach. Psychological Bulletin, 103(3), 411-423.

Bents, R.T., Tokish, J.M. \& Goldberg, L. (2004). Ephedrine, pseudoephedrine, and amphetamine prevalence in college hockey players: most report performance-enhancing use. PhysSportsmed, 32 (9), 30-34.

Bodin, D. \& Gonzalez, T. (2010). Se doperoupas. Prévenir le dopaje chez les jeunessportifs en Espagne et en France: approchemultidimensionnelle des processus de conduites dopantes. Université de Rennes 2, Faculte des sciences du Sport \& Universidad politécnica de Madrid, INEF Madrid. Project fundedby
WADA'S Social ScienceResearch. Accedido el 8 de enero, de 2015 desdehttps://www.wada-ama.org/en/resources/social-science/

Bollen, K.A. \& Long, J.S. (1993). Testing Structural Equation Models. Sage: Newbury Park, CA.

Bondarev, D., Galchinskiy, V., Ajitskiy, C. \& Labskir, V. (2009). A study of surroundings influence on attitude towards and behaviour regarding doping among ukranian athletes and nonathletes.Sevastopol National Technical University, Ukraine. Project funded by WADA'S Social Science Research. Accedido el 8 de enero, 2015 desde https://www.wada-ama.org/en/resources/socialscience/.

Boulu, R.G (2002). New provisions for prevention and fight against doping. Ann Pharm Fr, 60 (5), 291-295.

Burnkrant, R.E. \& Page, T.J.(1982). An examination of the convergent, discriminant and predictive validity of Fishbein's behavioral intention model. Journal of Marketing Research, 19 (4), 526-550.

Butryn, T., Johnson, J.A. \& Masucci, M.A. (2012). A qualitative examination of knowledge of doping, and motivations and/or deterrents to dope, among american and Canadian elite female triathletes. San Jose State University, USA. Project funded by WADA'S Social Science Research. Accedido el 15 de enero, 2015 desdehttps://www.wada-ama.org/en/resources/social-science/.

Byrne, B.M. (2001). Structural Equation Modeling with AMOS. Basic Concepts, Applications, and Programmin. Londres: LEA

Carmines, E.G. \& Zeller, R.A. (1979). Reability and Validity Assessment. Londres: Sage.

Carretero-Dios, H. \& Pérez, C. (2007). Standards for the development and the review of instrumental studies: Considerations about test selection in psychological research. International Journal of Clinical and Health Psychology, 7, 863-882.

Carretero-Dios, H. \& Pérez, C. (2005). Normas para el desarrollo y revisión de estudios instrumentales. International Journal of Clinical and Health Psychology, 5(3), 521-551.

Churchill, G.A. (1979). A Paradigm for Developing Better Measures of Marketing Constructs. Journal of Marketing Research, 16 (1), 6473.

Cordero, S.I., Cordero, A.T., Martínez, Y., Belkys, A. \& Cabrera, A. (2014). Juegos didácticos con enfoque creativo para contribuir a la educación antidoping en los atletas de fútbol, categoría 9-10 años. EFDeportes.com, Revista Digital. Buenos Aires, 193. Accedido el 25 de septiembre, 2014 desde http://www.efdeportes./com/

Cortina, J.M. (1993). What is coefficient alpha? An examination of theory and applications. Journal of Applied Psychology, 78, 98104.

Corvella, E. (2010). Análisis del conocimiento y la conciencia social del dopaje y la prevención entre los atletas en Córdoba (Argentina) y el enfoque preventivo. Becado por la AMA en 2008. Universidad Nacional de Córdoba. Argentina. Accedido el 15 de enero, 2015 desdehttps://www.wada-ama.org/en/resources/social-science/.

Dvorak, J., Saugy, M. \& Pitsiladis, Y.P. (2014). Los retos y amenazas a la aplicación de la lucha contra el dopaje en el deporte. Br Journal Sports Medicine, 48 (10), 807-809.

Elosua, P. (2003). Sobre la validez de los tests. Psicothema, 15(2), 315321.

Galán, I., Rodríguez-Aralejo, F., Díez-Gañán, A., Tobías, A., Zorrilla, B. \& Gandarillas A. (2006). Clustering of behavioural risk factors and compliance with clinical preventive recommendations in Spain. Preventive Medicine, 42 (5), 343-347

Giménez, F.J., Rodríguez, J.M. (2006). Buscando el deporte educativo ¿Cómo formar a los maestros?. Retos. Nuevas tendencias en Educación Física, Deportey Recreación, 9, 40-45.

Hair, J.F., Black, W.C., Babin, B.J., Anderson, R.E. \& Tatham, R.L. (2006). Multivariate data analysis (6 Edición). Upper Saddle River, NJ: Pearson-Prentice-Hall.

Heise, D.R. \& Bohrnstedt, G.W. (1970). Validity, Invalidity and Reliability. En Borgatta E.F. \& Bohrnstedt, G (Eds.), Sociological methodology (104-129). San Francisco: Jossey Bass. 
Laure, P., Lecerf, T., Friser, A., Binsinger, C., Lazuras, L. \& Barkoukis, V. (2004). Drugs, recreational drug use and attitudes towards doping of high school athletes. Int J Sports Med, 25 (2), 133-138.

Laure, P. \& Binsinger, C. (2005). Adolescent athletes and the demand and supply of drugs to improve their performance. J Sports Sci Med, 4 (3), 272-277.

Lazuras, L., Barkoukis, V. \& Tsorbatzoudis, H. (2015). Toward an integrative model of doping use: an empirical study with adolescent athletes. J Sport Exerc Psychol, 37 (1), 333-336.

Lehmann, D.R., Gupta, S., \& Steckel, J.H. (1999).Marketing Research. New York: Addison-Wesley.

López Pastor, V.M., Pérez Brunicardi, D., Manrique Arribas, J.C. \& Siglo XXI. Retos. Nuevas tendencias en Educación Física, Deporte y Recreación. 29, 182-187.

Lorente, F. O., Peretti-Watel, P. \& Grelot, L. (2005). Cannabis use to enhance sportive and non-sportive performances among French sports students. Addictive Behaviours, 30 (7), 1382-1391.

Marôco, J. (2010). Análise de Equações Estructurais. Fundamentos teóricos, Software \& Aplicações. Pero Pinheiro: Rolo y Filhos II, SA.

Méndez Alonso, D., Fernández Rio, J., Méndez Giménez, A. \& Prieto Saborit, J.A. (2016).Análisis de los currículos autonómicos LOMCE de Educación Física en Educación Primaria. Retos. Nuevas tendencias en Educación Física, Deporte y Recreación. 28, 15-20.

Melia, P., Pipe, A. \& Greenberg, L. (1996). The use of anabolicandrogenic steroids by Canadian students. Clin J Sport Med. 6(1), 9-14.

Naylor,A.H., Gardner, D. \& Zaichkowsky, L. (2001). Drug use patterns among high school athletes and nonathletes. Adolescence. 36(144): 627-639.

Nunnally, J.C. \& Bernstein, I.J. (1995). Teoría psicométrica. Madrid: McGraw-Hill.

Ozdemir, L., Nur, N., Bagcivan, I., Bulut, O., Sümer, H. \& Tezeren, G. (2005). Doping and performance enhancing drug use in athletes living in sivas, mid-anatolia: a brief report. J Sports Sci Med, 4 (3), 248-252. Monjas Aguado, R. (2016). Los retos de la Educación Física en el

Peretti-Watel, P. \& Lorente, F.O. (2004). Cannabis use, sport practice and other leisure activities at the end of adolescence. Drug Alcohol Depend, 73 (3), 251-257.

Reardon, C., \& Creado, S., (2014). Drug abuse in athletes. Subst Abuse Rehabil, 14(5), 95-105.

Rodríguez, D. (2010). El fútbol como herramienta para el trabajo de los valores y actitudes en la ESO según las Competencias Básicas. Football as a tool for the job of the values and attitudes in the ESO according to the Basic Competencies. Retos. Nuevas tendencias en Educación Física, Deportey Recreación. 17, 67-71.

Rubio, D.M., Berg-Weger, M., Tebb, S.S., Lee, E.S. \& Rauch, S. (2003). Objectifying content validity: Conducting a content validity study in social work research. Social Work Research, 27, 94-104.

Ruiz Omeñaca, J.V., Ponce de León, A., Sanz, E. \& Valdemoros, M.A. (2015). La educación en valores desde el deporte: investigación sobre la aplicación de un programa integral en deportes de equipo. Retos. Nuevas tendencias en Educación Física, Deporte y Recreación. 28, 270-275.

Sánchez, D., Leo, F.M., Amado, D., González, I. \& López, J.M. (2012). Análisis diferencial de la percepción del desarrollo de valores en las clases de educación física. Differential Analysis of perception of the development of values in Physical Education clases. Retos. Nuevas tendencias en Educación Física, Deporte y Recreación. 22, 9-12.

Skinner, J., Moston, S. \& Engelberg, T. (2012). The relationship between moral code, participation in sport, and attitudes towards performance enhancing drugs in young people. University of Canberra, Australia. Project funded by WADA'S Social Science Research. Accedido el 8 de enero, de 2015 desde https://www.wada-ama.org/ en/resources/social-science/

Stilger, V.G. \& Yesalis, C.E. (1999). Anabolic androgenic steroid use among high school football players. J Community Health, 24 (2), 131-145.

Streiner, D.L. \& Norman, G.L. (1995). Health measurement scales: A practical guide to their development and use ( $2^{\mathrm{a}}$ ed.). New York: Oxford University Press.

World Antidoping Code. (2015). Accedido el 20 octubre, 2014, desde https://www.wada-ama.org/en/what-we-do/the-code.

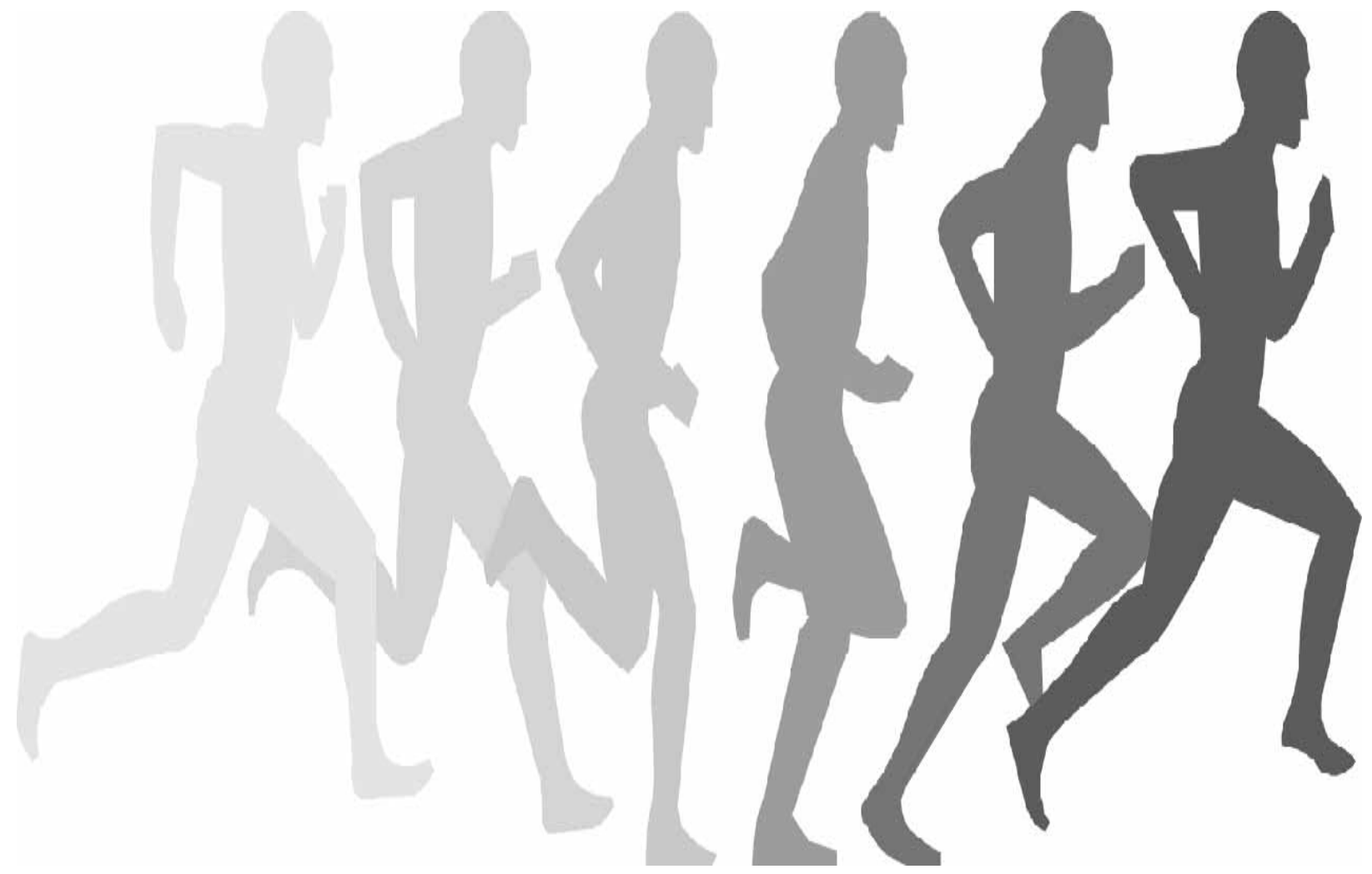

